\title{
Total arch repair with open triple-branched stent graft placement for acute type A aortic dissection: Experience with 122 patients
}

\author{
Liang-Wan Chen, MD, Lin Lu, MD, Xiao-Fu Dai, MD, Xi-Jie Wu, MD, Gui-Can Zhang, MD, \\ Guo-Feng Yang, MD, and Yi Dong, MD
}

Objective: To summarize the clinical experiences and midterm follow-up results of total arch repair with open triple-branched stent graft placement for acute type A aortic dissection.

\begin{abstract}
Methods: From June 2008 to March 2013, 122 patients (95 men and 27 women; mean age, $50.9 \pm 10.4$ years) with acute type A aortic dissection underwent total arch repair with open placement of a triple-branched stent graft under hypothermic cardiopulmonary bypass and selective cerebral perfusion. During the follow-up period, enhanced computed tomography and echocardiography were performed at 3 months postoperatively and annually thereafter.

Results: Placement of the triple-branched stent graft into the true lumen of the descending aorta, arch, and 3 arch vessels was technically successful in 121 patients. The cardiopulmonary bypass time was $186.50 \pm 38.23$ minutes, and the selective antegrade cerebral perfusion time was $31.97 \pm 10.08$ minutes. The in-hospital mortality was $4.93 \%$. No permanent neurologic dysfunction or paraplegia was observed. Three patients were lost to follow-up. The mean follow-up period was $30.24 \pm 12.35$ months. After hospital discharge, 3 patients died. On the 3-month postoperative scans, complete thrombus formation around the triple-branched stent graft was observed in $89.38 \%$ of the patients. Endoleaks were detected in 12 patients; 8 patients refused any management for the endoleaks, but they maintained a good quality of life. The other 4 patients were successfully treated by additional surgery.
\end{abstract}

Conclusions: Total arch repair with open triple-branched stent graft placement is an effective technique with satisfactory early and midterm results. This technique could be an attractive alternative to conventional total arch replacement. (J Thorac Cardiovasc Surg 2014;148:521-8)

Simple graft replacement of the ascending aorta is the conventional treatment of acute Stanford type A aortic dissection. ${ }^{1-3}$ However, residual dissection in the arch and downstream aorta after the conventional operation still occurs in $50 \%$ to $70 \%$ of patients, and it has been widely proved to play an essential role in determination of the long-term prognosis. ${ }^{4-7}$ This unsatisfactory long-term prognosis would favor the simultaneous replacement of the ascending aorta and total arch in the same surgical field during the primary emergency operation. ${ }^{8}$

Simultaneous replacement of the ascending aorta and total arch is a far more invasive and risky procedure than conventional ascending replacement for acute type A aortic dissection, mainly because it requires elaborate

\footnotetext{
From the Department of Cardiac Surgery, Union Hospital, Fujian Medical University, Fuzhou, Fujian, China.

This research was sponsored by Chinese national and Fujian provincial key clinical specialty construction programs.

Disclosures: Authors have nothing to disclose with regard to commercial support.

Received for publication July 30, 2013; revisions received Sept 20, 2013; accepted for publication Oct 14, 2013; available ahead of print Nov 25, 2013.

Address for reprints: Liang-Wan Chen, MD, Department of Cardiac Surgery, Union Hospital, Fujian Medical University, Fuzhou, Fujian 350001, China (E-mail: chenliangwan@tom.com).

$0022-5223 / \$ 36.00$

Copyright (C) 2014 by The American Association for Thoracic Surgery

http://dx.doi.org/10.1016/j.jtcvs.2013.10.021
}

anastomoses of the graft to the dissected and fragile descending aorta and 3 arch vessels. ${ }^{2}$ These time-consuming steps under emergency conditions could confer additional risks to the patients. Whether this total arch replacement with the possible additional operative risk could be justified from the viewpoint of the potential long-term benefits remains controversial. ${ }^{9,10}$

It would be desirable if 1 technique could be effectively used to repair the total arch while keeping the risk and surgical invasiveness as low as possible. Enlightened by the conventional endovascular straight stent graft technique for acute aortic dissection, ${ }^{11-13}$ we recently developed a simple, open, triple-branched stent graft (branched 1-piece stent graft) placement technique. ${ }^{14,15}$ Using our new technique, total arch repair can be performed simply by open placement of the triple-branched stent graft into the proximal descending aorta, arch, and 3 arch vessels, instead of direct surgical repair. In the present study, we retrospectively reviewed our experience and evaluated the effectiveness of total arch repair using this technique to repair acute type A aortic dissection.

\section{METHODS}

\section{Patients}

From June 2008 through March 2013, 122 patients with acute Stanford type A aortic dissection underwent total arch repair with open placement 
TABLE 1. Preoperative characteristics

\begin{tabular}{lc}
\hline \multicolumn{1}{c}{ Characteristic } & Value \\
\hline Patients (n) & 122 \\
Age (y) & \\
$\quad$ Mean \pm standard deviation & $50.9 \pm 10.4$ \\
$\quad$ Range & $24-73$ \\
Gender (n) & \\
$\quad$ Male & 95 \\
Female & 27 \\
Hypertension (n) & 93 \\
Diabetes (n) & 11 \\
Chronic renal dysfunction (n) & 3 \\
Cardiac tamponade (n) & 5 \\
Moderate or severe aortic valve regurgitation (n) & 39 \\
Acute left heart failure (n) & 2 \\
Transient brain ischemia (n) & 2 \\
Acute renal dysfunction (n) & 7 \\
Transient right leg ischemia (n) & 1 \\
\hline
\end{tabular}

using the triple-branched stent graft technique. The ethics committee of our hospital approved this experimental procedure, and each patient or legal representative provided written informed consent. The diagnosis was made on the basis of electron beam computed tomography, magnetic resonance imaging, and echocardiography. The primary intimal tears were located in the ascending aorta in 69 patients, the arch in 11 patients, and the proximal descending aorta with retrograde extension of the dissection into the arch and ascending aorta in 42 patients. The innominate artery was involved by the dissection in 71 patients, and the dissected innominate artery with a smaller real lumen and larger false lumen was observed in 24 patients. The left common carotid artery was involved by the dissection in 17 patients, and the left subclavian artery was involved in 4 patents. All those with involved left common carotid arteries and left subclavian arteries had a larger real lumen and smaller false lumen. The preoperative characteristics are listed in Table 1. The arch vessel diameter at its base, arch size at the base of the innominate artery, and aortic diameter of the middle thoracic descending aorta were recorded (Table 2). The interval from the onset of pain to surgery varied from 1 to 13 days (average, $5.27 \pm 3.19$ ). All operations were performed within 4 hours after the diagnosis had been confirmed.

The 122 patients had 1 of the following total arch repair indications: (1) age $<55$ years, (2) an intimal tear located in the transverse arch or proximal descending aorta that could not be resected by hemiarch replacement, or (3) serious involvement of the arch vessels. These patients were selected for the open triple-branched stent graft placement technique because the native aortic arch diameter, arch vessel diameter, and distances between 2 neighboring arch vessels matched the corresponding sizes of the triple-branched stent graft available. The diameter of the native aortic arch and arch vessels was $10 \%$ to $20 \%$ smaller than the diameter of the corresponding stent grafts, and a slight difference $(<20 \%)$ between the distance of 2 neighboring arch vessels and the distance of the 2 corresponding

TABLE 2. Diameter of native vessels and aortic segments

\begin{tabular}{lcc}
\hline \multicolumn{1}{c}{ Variable } & Mean \pm SD $(\mathbf{m m})$ & Range $(\mathbf{m m})$ \\
\hline Innominate artery & $14.93 \pm 4.5$ & $11-20$ \\
Left common carotid artery & $9.16 \pm 1.1$ & $8-12$ \\
Left subclavian artery & $10.63 \pm 1.2$ & $8.9-12.7$ \\
Proximal arch & $28.9 \pm 3.2$ & $23-31$ \\
Middle thoracic descending aorta & $23.7 \pm 2.9$ & $22-25$ \\
\hline
\end{tabular}

$S D$, Standard deviation.
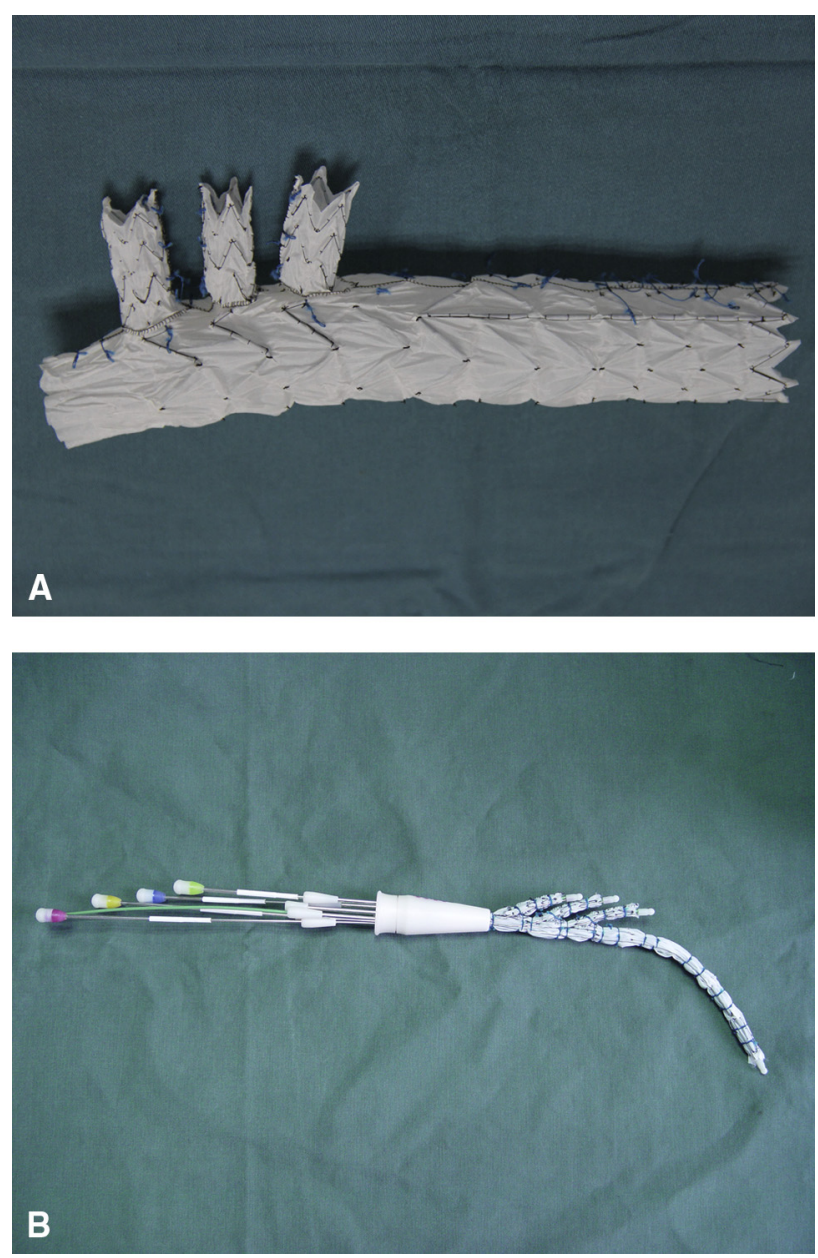

FIGURE 1. A, The triple-branched stent graft included a main stent graft and 3 sidearm stent grafts. B, The main stent graft and 3 sidearm stent grafts were individually mounted on 4 catheters and restrained by 4 silk sutures.

sidearm stent grafts was acceptable. The preoperative and intraoperative exclusion criteria for this procedure were as follows: a history of carotid artery disease, acute type A aortic dissection with an arch or a proximal descending aortic aneurysm, and all 3 arch branch ostia could not been seen clearly from the arch true lumen through the transverse incision of the distal ascending aorta.

During the same 5-year study period, 57 patients with acute type A dissection who were excluded from total arch repair underwent conventional graft replacement of the ascending aorta or ascending aorta and proximal arch. In another 46 patients with an indication for total arch repair but who could not satisfy the inclusion and exclusion criteria for open placement of a triple-branched stent graft, total arch replacement using the conventional technique was performed.

\section{Triple-Branched Stent Graft Description}

The triple-branched stent graft was a branched 1-piece graft and included a main stent graft and 3 sidearm stent grafts (Yuhengjia Science and Technology, Corp, Ltd, Beijing, China). Each stent graft consisted of a self- expandable nitinol stent and polyester vascular graft fabric. The main tube graft was $145 \mathrm{~mm}$ long, 30 or $32 \mathrm{~mm}$ in the proximal diameter, and 26 or $28 \mathrm{~mm}$ in the distal diameter. The first sidearm graft was $35 \mathrm{~mm}$ long and 14 or $16 \mathrm{~mm}$ in diameter. The second and third sidearm grafts were both $25 \mathrm{~mm}$ long and 12 or $14 \mathrm{~mm}$ in diameter. The distance between 

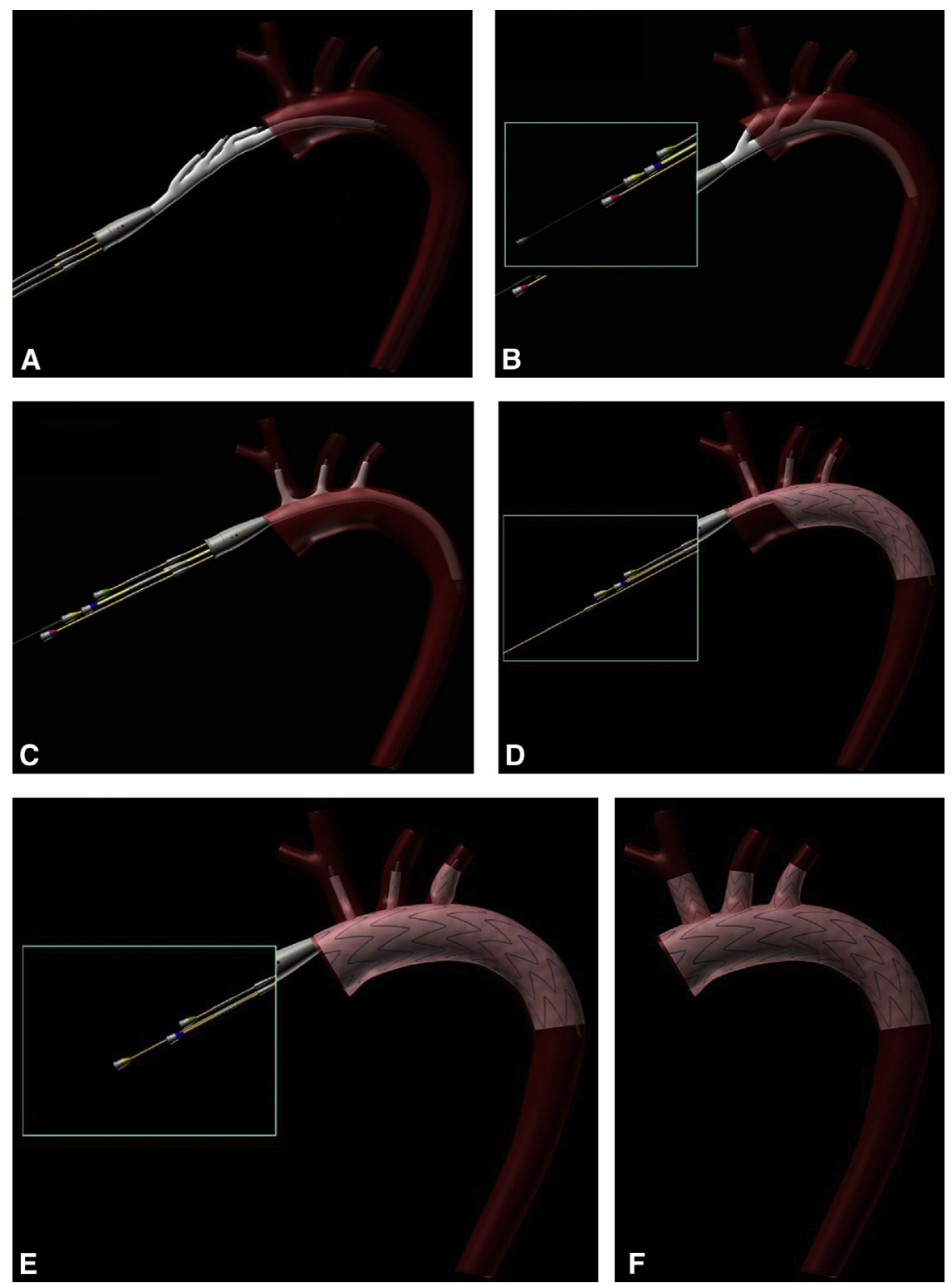

FIGURE 2. Schematic diagram of the operation. A, Through this distal ascending aortic transverse incision, the main stent graft was directly inserted into the true lumen of the arch and proximal descending aorta. B, Next, each sidearm stent graft was implanted 1 by 1 into the corresponding arch vessel. C, After the main stent graft and sidearm stent grafts were correctly placed, D-F, the triple-branched stent graft was deployed by withdrawing the restraining sutures.

the 2 neighboring sidearm grafts was $3 \mathrm{~mm}$. The main stent graft and sidearm stent grafts were individually mounted on 4 catheters and restrained by 4 silk sutures (Figure 1). This triple-branched stent graft has been previously described in detail by our research group. ${ }^{14,15}$

\section{Procedure}

The details of the procedure have been previously reported. ${ }^{14,15}$ It was performed in all patients by way of a median sternotomy under cardiopulmonary bypass with selective cerebral perfusion. The right axillary artery was routinely used as the arterial return cannula for cardiopulmonary bypass and selective cerebral perfusion. Cardiopulmonary bypass flow was maintained at 2.4 to $2.6 \mathrm{~L} / \mathrm{min} / \mathrm{m}^{2}$. During core cooling, the innominate and left common carotid arteries were dissociated from the surrounding tissue. Aortic root manipulation, such as aortic valve repair and sinus of Valsalva reconstruction, was performed after distal ascending aortic crossclamping and cardioplegia. Next, a straight or single-branched Dacron tube graft (Boston Scientific, Inc, Boston, Mass) was anastomosed to the repaired aortic root.

When the rectal temperature reached $22^{\circ} \mathrm{C}$, selective cerebral perfusion was started at a rate of 10 to $15 \mathrm{ml} / \mathrm{kg} / \mathrm{min}$. The innominate and left common carotid arteries were crossclamped, and the distal ascending aorta was transected at the base of the innominate artery. Through this distal ascending aortic transverse incision, the main stent graft was directly inserted into the true lumen of the arch and proximal descending aorta (Figure 2, A), and then each sidearm stent graft was implanted 1 by 1 into the corresponding arch vessel (Figure 2,B). After the main stent graft and sidearm stent grafts had been correctly placed (Figure 2, C), the triple-branched stent graft was deployed by withdrawing the restraining sutures (Figure 2, $D$ to $F$ ) and dilated with balloon catheters to confirm full opening. From August 2011, to avoid endoleak from the innominate 
arterial branch, we began applying innominate arterial banding at its base for the dissected innominate artery after the triple-branched stent graft had been successfully inserted and deployed. The banding felt is a Dacron tube graft ring $3 \mathrm{~mm}$ in width and $5 \%$ to $10 \%$ shorter than the size of the implanted sidearm graft in length.

Finally, the distal aorta incorporating the triple-branched stent graft was directly anastomosed to the distal end of the straight or 1-branched Dacron tube graft. Next, antegrade systemic perfusion from the Dacron tube graft was resumed and rewarming started.

\section{Follow-up Data}

After hospital discharge, the patients were followed up to the end of the study period (March 2013) or death. They were interviewed by telephone or direct interviews in our department. Three patients were lost to follow-up. The follow-up period ranged from 2 to 51 months (mean, $30.247 \pm 12.35$ ).

During the follow-up period, the patients underwent contrast-enhanced computed tomography and echocardiography as follows: before discharge, 3 months postoperatively, and annually thereafter. The effectiveness of the open triple-branched stent graft placement was estimated by complete thrombus obliteration of the false lumen surrounding the triple-branched stent graft.

\section{RESULTS \\ Operative Data}

Placement of the triple-branched stent graft into the true lumen of the proximal descending aorta, arch, and 3 arch vessels could be easily completed within 3 to 6 minutes. In the present series, placement of the triple-branched stent graft was technically successful in 121 of 122 patients $(99.18 \%)$. All placed triple-branched stent grafts in those 121 patients had a good position and wide expansion found on the postoperative chest radiographs or computed tomography scans. Only 1 placement failure occurred: the sidearm stent graft had slipped from the orifice of the left subclavian artery into the true lumen of the proximal descending aorta. The mean cardiopulmonary bypass time was $186.50 \pm 38.23$ minutes (range, 122-389), the aortic crossclamp time was $78.28 \pm 21.06$ minutes (range, 25-150), and the selective cerebral perfusion and lower body arrest time was $31.97 \pm 10.08$ minutes (range, 15-76). Simultaneous with triple-branched stent graft placement, 19 patients underwent the Bentall procedure, 64 underwent aortic root reconstruction, 23 underwent aortic valve repair, and 3 underwent coronary artery bypass grafting.

\section{Early Mortality and Morbidity}

The 30-day operative mortality was $3.28 \%$ (4 of 122). Two patients with preoperative acute renal failure died of multiorgan failure. One patient, who had developed preoperative pericardial tamponade and had been resuscitated before the establishment of cardiopulmonary bypass, died of heart failure. The fourth patient died of acute respiratory distress syndrome and mesenteric ischemia. Two other patients died in the hospital, for an overall in-hospital mortality rate of $4.93 \%$ (6 of 122). One patient with preoperative acute renal dysfunction died of massive gastrointestinal bleeding during postoperative blood dialysis. The second patient, with preoperative pneumonia, required continuous postoperative mechanical ventilator support and tracheotomy, and died of multiorgan failure.

The postoperative cerebral complications included cerebral infarction in 2 patients and global temporary neurologic dysfunction in 11 patients. However, they had recovered fully before hospital discharge. Ventilator support for $>5$ days was required in 9 patients, and a tracheotomy was required in 5 patients. Acute renal failure complicated the postoperative care for 11 patients, with 9 requiring dialysis. Postoperative pericardial effusions requiring drainage developed in 17 patients and mediastinitis in 1 patient. No patient required repeat surgery to correct excessive postprocedural bleeding.

\section{Follow-up Data}

During the follow-up period, 3 patients died. One patient died of sudden chest pain at home at 7 months postoperatively. The second patient, who had undergone aortic valvular repair during the primary surgery, died of severe aortic valvular insufficiency at 42 months postoperatively. The postoperative severe aortic valvular insufficiency had been found at 6 months after surgery, and the patient had refused reoperation to treat the aortic valve. The third patient, who had had chronic renal failure preoperatively, died of pneumonia at 14 months postoperatively. All the survivors had resumed normal activities.

\section{Computed Tomography and Echocardiography}

Follow-up computed tomography and echocardiography were performed according to schedule in 113 patients. At the 3-month postoperative scans, complete thrombus formation around the triple-branched stent graft was observed in 101 of the 113 patients $(89.38 \%)$, at the diaphragmatic level in $71(62.83 \%)$, and at the level of the superior mesenteric artery in 5 patients $(4.42 \%$; Figure $3, A$ ). Although residual dissection in the abdominal aorta occurred in most patients, obvious enlargement of the residual dissected aorta was not found compared with the preoperative imaging findings. In the aortic segment with complete thrombus formation of the false lumen, a continuous aortic healing process was noted during the follow-up period, including absorption of the false lumen thrombosis, enlargement of the true lumen, and shrinkage of the aortic diameter to the normal aortic size (Figure 3,B). Creation of a new intimal tear by the edge of the stent graft was not observed in any case. No sidearm stent graft stenosis or occlusion was found; however, a slight increase in flow velocity $(<2.5 \mathrm{~m} / \mathrm{s})$ in 2 sidearm grafts for the innominate arteries was noted. During 

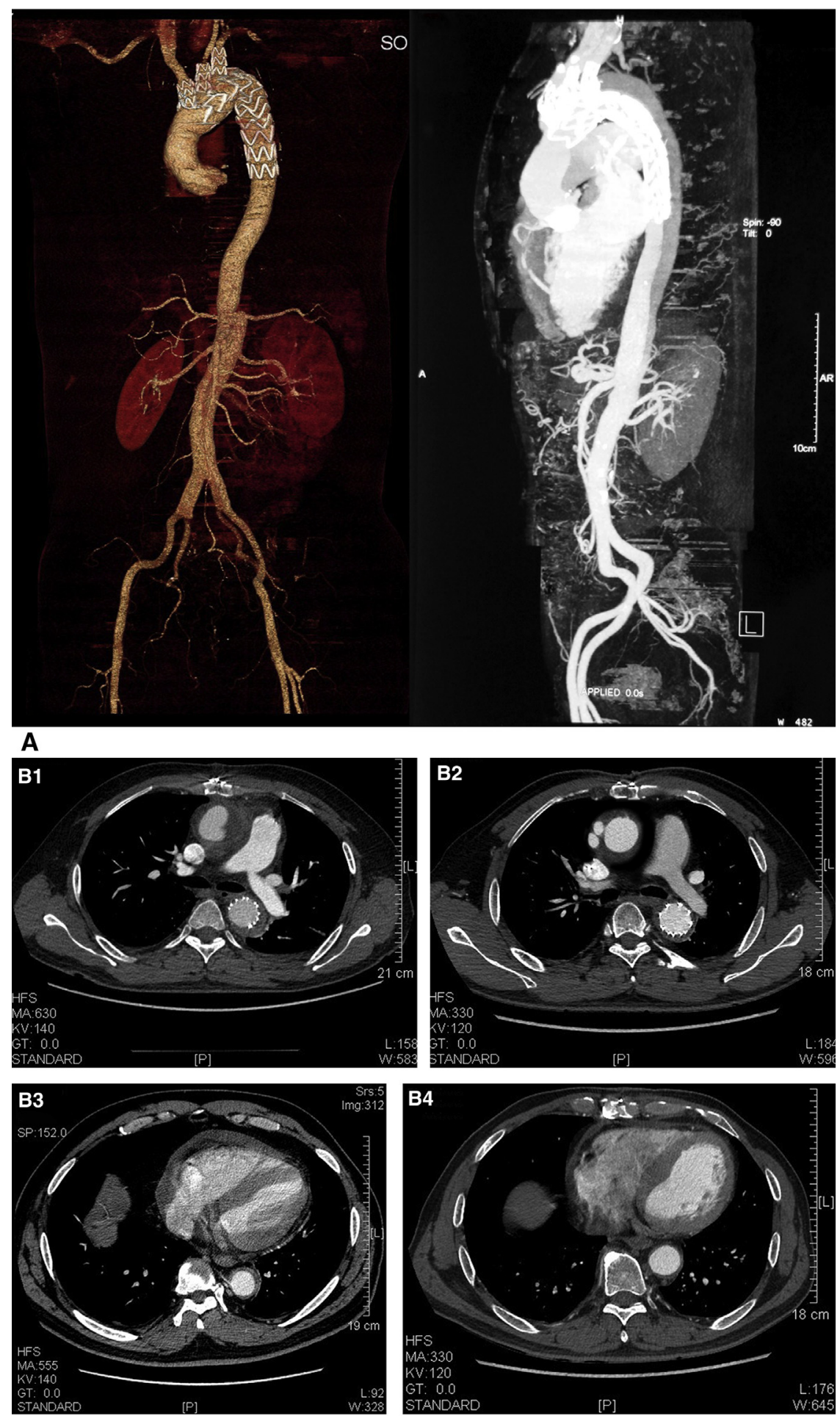

B

FIGURE 3. Postoperative results on computed tomography images. A, The false lumen of the arch and descending aorta was completely closed by postoperative thrombus formation. B, A patient with typical aortic remodeling showing complete thrombus formation B1, in the false lumen around the stent graft and B2, at the diaphragmatic level 2 weeks after surgery. One year later, the false channel thrombosis had been nearly reabsorbed B3, around the stent graft and B4, at the diaphragmatic level. C, Innominate endoleak. D, A patient with innominate banding (D1, preoperatively; D2, postoperatively). 


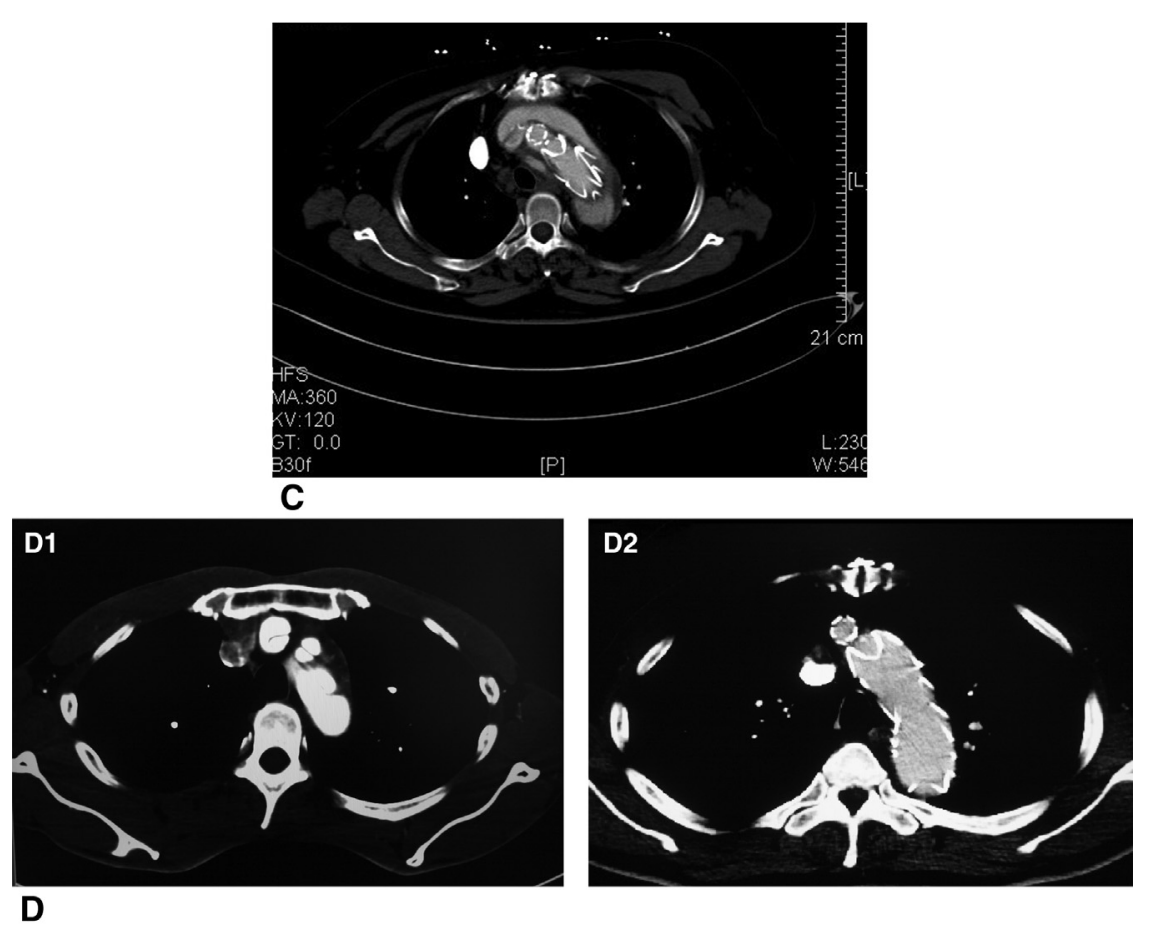

FIGURE 3. (Continued).

the follow-up period, the flow velocity did not increase further.

The distal ends of the main stent grafts were located between the T7 and T8 levels in 93 patients. No any distal end below the T8 level was found.

\section{Endoleak Development}

In the present series, endoleaks were detected in 12 patients, including the patient with triple-branched stent graft placement failure. Leakage from the suture site of the main stent graft was found in 1 patient. The other 10 endoleaks had arisen from the distal ends of the sidearm stent grafts inside the innominate arteries (Figure 3,C). A dissected innominate artery with the size of the false lumen much larger than that of the true lumen was found on preoperative imaging for all 10 of these patients. However, since 2011, when the banding technique began to be used for the dissected innominate arteries, no endoleak from the innominate arterial branch has been observed (Figure 3,D).

Of the 12 patients with endoleaks, 8 with endoleaks from the innominate arterial branches refused any treatment of the endoleaks. However, they maintained a good quality of life. The other 4 patients underwent surgical management for the endoleaks successfully. The patient with placement failure underwent reimplantation of the triple-branched stent graft under hypothermic circulatory arrest and selective antegrade cerebral perfusion. In the patient with endoleak from the suture site of the main stent graft, we found that the continuous suture did not incorporate the main stent graft for the last 2 stitches. During reoperation, the free margin of the main stent graft was directly fixed to the anastomosis site. For 2 patients with endoleaks from the innominate arterial branches, the innominate arterial roots containing the sidearm stent grafts were closed, and innominate arterial grafting from the Dacron tube, which had replaced the ascending aorta in the primary procedure, was performed under extracorporeal circulation. After surgical management of the endoleaks, the false lumen in the arch and the proximal descending aorta were no longer detected in those 4 patients.

\section{DISCUSSION}

Our open triple-branched stent graft placement technique was designed to repair the total arch by the simple open placement of triple-branched stent graft instead of direct surgical repair. ${ }^{14,15}$ In our series, thrombus obliteration of the residual false lumen in the arch and proximal descending thoracic aorta, where the stent graft had been implanted, was observed in $89.38 \%$ of the patients. This result suggests that our triple-branched stent graft placement technique can effectively repair the total arch.

In total arch replacement for acute type A aortic dissection, the elephant trunk technique has been routinely used to achieve a stronger distal anastomosis and to facilitate subsequent surgery on the distal aorta. ${ }^{16,17}$ However, placing the prosthetic graft into the true lumen of the dissected descending aorta is not easy, and some 
complications, resulting from the "flapping" action of the prosthetic graft, such as kinking and obstruction of the graft, embolization, and paraplegia, have been observed. ${ }^{18}$ To avoid such problems from the conventional elephant trunk technique, some surgeons have recently developed a stented elephant trunk technique in which a straight stent graft is implanted into the descending aorta during total arch replacement. ${ }^{11,12,19}$ This stented elephant trunk has been proved to provide an effective method of closing the residual false lumen of the downstream descending aorta, which might contribute to better long-term outcomes for acute type A aortic dissection. ${ }^{11,12,19}$ Therefore, this stented elephant trunk technique has been accepted as the preferred surgical treatment of acute type A aortic dissection when total arch replacement is performed. Our triple-branched stent graft includes a main stent graft and 3 sidearm stent grafts. The proximal part of the main stent graft provided effective repair of the total arch and the distal part acted as the stented elephant trunk. Therefore, both the scope of the repaired thoracic aorta and the short-term outcome of the residual false lumen with our triple-branched stent graft placement technique should be comparable to that of traditional total arch replacement combined with open placement of a stented elephant trunk graft.

With conventional ascending aorta replacement, the distal aortic anastomosis is frequently performed at the dissected site. After acute dissection, the aortic tissues are friable and of poor quality; therefore, anastomosis between a dissected aortic stump and a Dacron tube graft often results in intraoperative or postoperative hemorrhage owing to tissue tearing at the suture line. ${ }^{16}$ Reinforcement of the dissected aortic stump has been routinely performed to secure good hemostasis at the suture line. In our technique, although the distal aortic stump incorporating the stent graft was directly anastomosed to the Dacron tube without any other reinforcement, no problems occurred with bleeding from the distal aortic anastomosis either intraoperatively or postoperatively. This has shown that our triplebranched stent graft provides a strong distal aortic stump to achieve hemostasis. The implanted stent graft closed the false lumen, approximated the dissected layers, and, consequently, interrupted back flow from the false lumen, which can be a source of bleeding at the anastomosis. Furthermore, in the anastomosis of the Dacron tube to the distal aortic stump with incorporation of the stent graft, the stent graft acted as the neointima at the suture line. This stent graft neointima effectively prevented anastomotic blood leakage from a small tear at the suture line.

In our technique, total arch repair could be easily completed by inserting the triple-branched stent graft into the proximal descending aorta, arch, and 3 arch vessels through a transverse incision of the distal ascending aorta during deep hypothermic circulatory arrest. This distal aortic incision was the same as that used in conventional ascending graft replacement. Although insertion of the triple-branched stent graft required 3 to 6 minutes, the cardiopulmonary bypass time, aortic crossclamp time, and selective cerebral perfusion and lower body arrest time in our series were comparable to those for conventional ascending replacement with open distal aortic anastomosis. This was because our triple-branched stent graft provided a strong distal aortic stump, obviating the need for distal aortic stump reinforcement. Therefore, our technique could reduce the risk and technical difficulty of total arch repair to close to that of conventional ascending graft replacement with open distal anastomosis. In the present series, the in-hospital mortality was $4.93 \%$. This good result seemed to be because our open triple-branched stent graft technique reduced the surgical invasiveness.

The proper size of the implanted triple-branched stent graft was very important, not only for dilation of the true lumen, shrinkage, and quick clot formation of the false lumen, but also for preventing new intimal trauma resulting from continuous compression of the oversized stent graft on the dissected and friable intimal wall. The diameter of the implanted triple-branched stent graft should be $10 \%$ to $20 \%$ larger than the diameter of the native aortic arch and arch vessels. ${ }^{20,21}$ Theoretically, the distance between the 2 neighboring sidearm stent grafts should be equal to the distance between the 2 corresponding arch vessels, which would keep the sidearm stent grafts from being twisted or kinking after deployment. However, in our practice, we found that a slight difference $(<20 \%)$ between those 2 diameters was acceptable. Two major reasons might have contributed to this finding. First, the main stent graft had some flexibility and bendability. Second, the existence of a small angulation between the main stent graft and sidearm graft did not result in distortion of the sidearm stent graft.

Endoleak development was the main factor affecting the efficacy of the triple-branched stent graft placement technique for acute type A aortic dissection. A total of 12 endoleaks occurred in our series, and most were identified from the innominate arterial branches. In the patients with endoleaks from the innominate arterial branches, preoperative computed tomography revealed that all those innominate arteries had been involved by the dissection and the size of the false lumen had been much larger than that of the true lumen. An incorrect size of the selected sidearm stent graft for those innominate arteries might have been the main factor contributing to endoleak development. In our series, the innominate artery was much more frequently affected by dissection than either the left common carotid or the subclavian artery. For a dissected innominate artery with a large false lumen and small real lumen, it was very difficult to determine the proper size of the sidearm stent graft during surgery. 
However, endoleak prevention in such cases became easier using innominate arterial banding. Once the innominate arterial banding technique was routinely applied to the dissected innominate arteries, we did not encounter any endoleak from the innominate arterial branch. Of the 12 patients with endoleaks, 4 underwent successful surgical management for the endoleaks. After surgical management for the endoleaks, the false lumen in the arch and the proximal descending aorta were no longer detected in those 4 patients, indicating that a secondary operation could be effective for repairing postoperative endoleaks.

The primary limitation of the present study was that comparisons between our open triple-branched stent graft placement group and conventional ascending replacement group or total arch replacement with stented elephant trunk group were not made. Although the preliminary outcomes were encouraging, with a mean follow-up period of 30.24 \pm 12.35 months, it is necessary to evaluate the long-term outcomes of this new technique for acute type A aortic dissection.

\section{References}

1. Fuster V, Ip JF. Medical aspects of acute aortic dissection. Semin Thorac Cardiovasc Surg. 1991;3:219-24.

2. DeSanctis RW, Doroghazi RM, Austen WG, Buckley MJ. Aortic dissection. N Engl J Med. 1987;317:1060-7.

3. DeBakey ME, McCollum CH, Crawford ES, Morris GC Jr, Howell J, Noon GP, et al. Dissection and dissecting aneurysms of the aorta: twenty-year follow-up of five hundred twenty-seven patients treated surgically. Surgery. 1982;92:1118-34.

4. Fann JI, Smith JA, Miller DC, Mitchelll RS, Moore KA, Grunkemeier G, et al. Surgical management of aortic dissection during a 30-year period. Circulation. 1995;92(9 Suppl):II113-21.

5. Haverich A, Miller DC, Scott WC, Mitchell RS, Oyer PE, Stinsion EB, et al. Acute and chronic aortic dissections-determinants of long-term outcome for operative survivors. Circulation. 1985;72:1122-34.

6. Ergin MA, Philips RA, Galla JD, Lansman SL, Mendelson DS, Quintana CS, et al. Significance of distal false lumen after type A dissection repair. Ann Thorac Surg. 1994;57:820-5.

7. Bachet JE, Termignon JL, Dreyfus G, Goudot B, Martinelli L, Piquois A, et al. Aortic dissection: prevalence, cause, and results of late reoperations. $J$ Thorac Cardiovasc Surg. 1994;108:199-206.
8. Ochiai Y, Imoto Y, Sakamoto M, Ueno Y, Sano T, Baba H, et al. Long-term effectiveness of total arch replacement for type A aortic dissection. Ann Thorac Surg. 2005;80:1297-302.

9. Massimo CG, Presenti LF, Marranci P, Favi PP, Poma AG, Ponzalli M, et al. Extended and total aortic resection in the surgical treatment of acute type A aortic dissection: experience with 54 patients. Ann Thorac Surg. 1988;46: 420-4.

10. Minale C, Splittgerber FH, Reifschneider HJ. Replacement of the entire thoracic aorta in a single stage. Ann Thorac Surg. 1994;57:850-5.

11. Liu ZG, Sun LZ, Chang Q, Zhu JM, Dong C, Yu CT, et al. Should the "elephant trunk" be skeletonized? Total arch replacement combined with stented elephant trunk implantation for Stanford type A aortic dissection. J Thorac Cardiovasc Surg. 2006;131:107-13.

12. Shimamura K, Kuratani T, Matsumiya G, Kato M, Shirakawa Y, Takano H, et al. Long-term results of the open stent-grafting technique for extended aortic arch disease. J Thorac Cardiovasc Surg. 2008;135:1261-9.

13. Nienaber CA, Fattori R, Lund G, Dieckmann C, Wolf W, Kodolitsch Y, et al. Nonsurgical reconstruction of thoracic aortic dissection by stent-graft placement. N Engl J Med. 1999;340:1539-45.

14. Chen LW, Dai XF, Zhang GZ, Lu L. Total aortic arch reconstruction with open placement of triple-branched stent graft for acute type A dissection. J Thorac Cardiovasc Surg. 2010;139:1654-5.

15. Chen LW, Dai XF, Zhang GZ, Cao H. Extensive primary repair of the thoracic aorta in acute type A aortic dissection by means of ascending aorta replacement combined with open placement of triple-branched stent graft: early results. Circulation. 2010;122:1373-8.

16. Mori Y, Hirose H, Takagi H, Umeda T, Fukumoto Y, Shimabukuro K, et al. Aortic arch repair for Stanford type A aortic dissection with distal anastomosis to the proximal level of the distal aortic arch. J Thorac Cardiovasc Surg. 2003;126: 415-9.

17. Ando M, Takamoto S, Okita Y, Morota T, Matsukawa R, Kitamura S. Elephant trunk procedure for surgical treatment of aortic dissection. Ann Thorac Surg. 1998;66:82-7.

18. Crawford ES, Coselli JS, Svensson LG, Safi HJ, Hess KR. Diffuse aneurysmal disease (chronic aortic dissection, Marfan, and mega aorta syndromes) and multiple aneurysm: treatment by subtotal and total aortic replacement emphasizing the elephant trunk operation. Ann Surg. 1990;211: $521-37$.

19. Sun LZ, Qi RD, Chang Q, Zhu JM, Liu YM, Yu CT, et al. Surgery for acute type A dissection using total arch replacement combined with stented elephant trunk implantation: experience with 107 patients. J Thorac Cardiovasc Surg. 2009; 138:1358-62.

20. Kato M, Ohnishi K, Kaneko M, Ueda T, Kishi D, Mizushima T, et al. New graft-implanting method for thoracic aortic aneurysm or dissection with a stented graft. Circulation. 1996;94:188-93.

21. Kato M, Matsuda T, Kaneko M, Kuratani T, Mizushima T, Seo Y, et al. Outcomes of stent-graft treatment of false lumen in aortic dissection. Circulation. 1998;98: $305-12$. 